\title{
Langer-Giedion syndrome associated with congenital dural arterio-venous fistula
}

\author{
Leu, Severina ; Valavanis, Anton ; Baltsavias, Gerasimos
}

\begin{abstract}
Langer-Giedion syndrome (LGS) is a rare disease caused by deletion of chromosome 8q23.3q24.11. Clinical manifestations include among others multiple exostoses, short stature, intellectual disability, and typical facial dysmorphism. Dural arterio-venous shunts (DAVS) in the pediatric age are rare lesions, which have been classified into three types: dural sinus malformations (DSM), infantile type DAVS (IDAVS), and adult type DAVS (ADAVS). We report a case of a patient with a known LGS who was diagnosed with complex intracranial dural AV fistula at the age of 20. An association between LGS and intracranial dural AV fistulas has to our knowledge never been reported before.
\end{abstract}

DOI: https://doi.org/10.1007/s00381-014-2570-9

Posted at the Zurich Open Repository and Archive, University of Zurich ZORA URL: https://doi.org/10.5167/uzh-100533

Journal Article

Published Version

Originally published at:

Leu, Severina; Valavanis, Anton; Baltsavias, Gerasimos (2015). Langer-Giedion syndrome associated with congenital dural arterio-venous fistula. Child's Nervous System, 31(5):801-804.

DOI: https://doi.org/10.1007/s00381-014-2570-9 


\title{
Langer-Giedion syndrome associated with congenital dural arterio-venous fistula
}

\author{
Severina Leu • Anton Valavanis • Gerasimos Baltsavias
}

Received: 25 September 2014 / Accepted: 29 September 2014 / Published online: 8 October 2014

(C) Springer-Verlag Berlin Heidelberg 2014

\begin{abstract}
Langer-Giedion syndrome (LGS) is a rare disease caused by deletion of chromosome 8q23.3-q24.11. Clinical manifestations include among others multiple exostoses, short stature, intellectual disability, and typical facial dysmorphism. Dural arterio-venous shunts (DAVS) in the pediatric age are rare lesions, which have been classified into three types: dural sinus malformations (DSM), infantile type DAVS (IDAVS), and adult type DAVS (ADAVS). We report a case of a patient with a known LGS who was diagnosed with complex intracranial dural AV fistula at the age of 20. An association between LGS and intracranial dural AV fistulas has to our knowledge never been reported before.
\end{abstract}

Keywords Langer-Giedion syndrome ·

Tricho-rhino-phalangeal disease type II · Arterio-venous

fistula $\cdot$ Dural sinus malformation

\section{Introduction}

Langer-Giedion syndrome (LGS) is a rare congenital disease caused by a deletion of chromosome 8q23.3-q24.11. It is also known as tricho-rhino-phalangeal disease type II (TRP II) and includes characteristics such as multiple exostoses, short stature, microcephaly, and facial dysmorphism $[1,2]$.

Dural AV shunts are shunts within the dura, typically at the wall of the venous sinuses. The clinical manifestation depends mostly on venous drainage pattern and location of the shunt [3]. They are commonly supplied by branches of dural arteries, sometimes from osseous branches and rarely from pial

S. Leu $(\bowtie) \cdot$ A. Valavanis $\cdot$ G. Baltsavias

Department of Neuroradiology, University Hospital of Zurich,

Rämistrasse 100, 8091 Zürich, Switzerland

e-mail: severina.leu@unibas.ch arteries. Drainage may be into the sinuses, osteodural veins, or retrograde into the cerebral veins [3].

\section{Case report}

A 20-year-old patient earlier diagnosed with sporadical Langer-Giedion syndrome showing a typical deletion of $8 \mathrm{q} 24$ presented with a first generalized epileptic seizure in 2012. Imaging workup revealed a complex intracranial AV fistula with multiple dilated cortical veins and signs of edema with venous congestion mostly in the left temporal lobe (Fig. 1). The patient developed exophthalmus of the left eye and complained of headaches. No tinnitus was referred. He had a short stature, facial dysmorphism, baldness, exostoses, syndactyly of the toes on the left foot, and his head circumference was above 97th percentile.

He was living with his parents and was able to practice an adapted job in daily life.

In the catheter angiography, the detailed angioarchitecture of the lesion was studied (Fig. 2). All potential dural arteries from the external and internal carotid and the vertebral arteries bilaterally were feeding the shunt, converging to a pouch of the proximal right sigmoid sinus. One of the main feeders was a persistent primitive trigeminal artery on the right side, arising from the basilar artery and supplying the shunt through its connection to the right middle meningeal artery. The venous drainage was anterograde to the ipsilateral internal jugular vein but mostly retrograde to the superior sagittal sinus and superficial convexial venous system, to the straight sinus and deep venous system and to the contralateral transverse sinus and through a remarkably dilated and tortuous Labbé vein to the sylvian vein and left cavernous sinus. The cavernous sinus was drained mainly through a dilated superior ophthalmic vein, which was responsible for the exophthalmus. 
Fig. 1 T2-weighted magnetic resonance (MR) images and time of flight angiography of a complex dural AV fistula at the level of the right transversesigmoid sinus junction with drainage through the left ophthalmic vein with accompanying exophthalmus
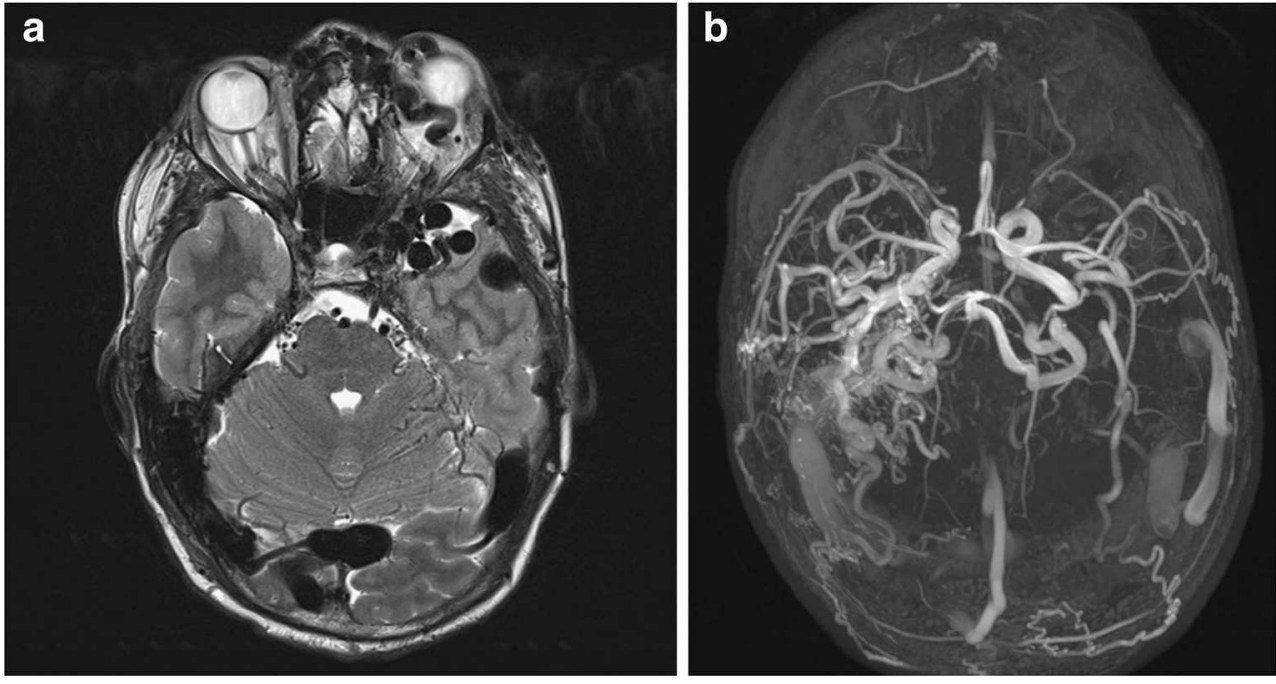

Endovascular treatment was performed in two stages: one stage with transarterial glue partial embolization for reduction of overall flow to the long-lasting congested cortical and orbital venous system and the second with transvenous approach, which resulted to a complete occlusion of the shunt. At the time between the two sessions, a small bleeding in the left temporal pole occurred without any focal neurologic deficit.
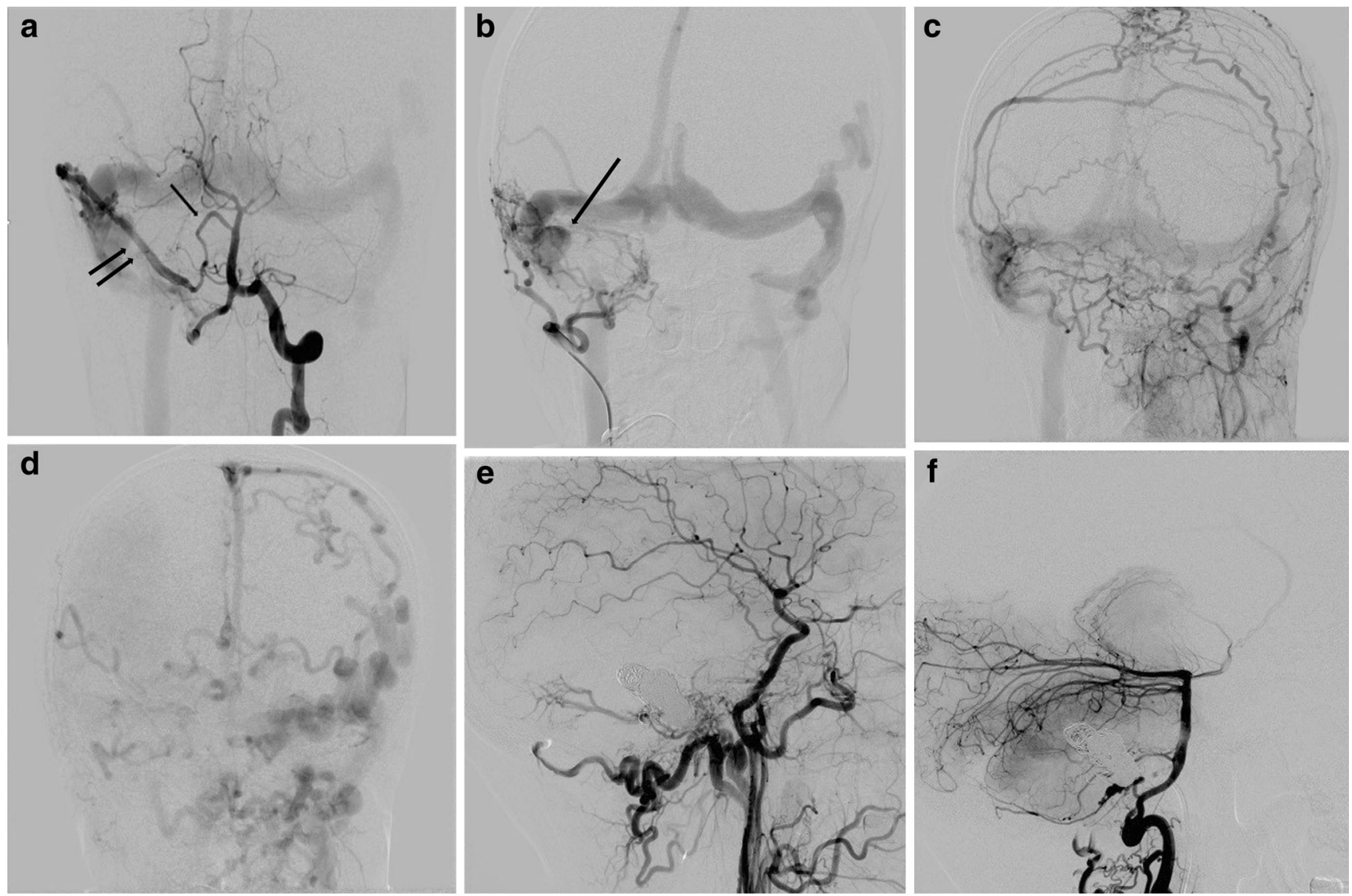

Fig. 2 Conventional angiography showing the complex AV fistula. a. Vertebral injection with demonstration of a right persistent trigeminal artery (arrow) supplying the fistula through its connection to the middle meningeal artery (double arrow). b. Right external carotid injection showing the convergence of the feeders into a venous pouch of the proximal sigmoid sinus (arrow). c. Left external carotid

injection showing all dural branches crossing the midline and converging to the fistula's venous pouch. d. Venous phase of a left common carotid injection showing the leptomeningeal venous drainage with dilation of cortical veins. e. Right common carotid, and $\mathbf{f}$. vertebral injection immediately postoperatively showing the complete obliteration of the shunt 


\section{Discussion}

\section{Dural AV shunts (DAVS)}

Based on Lasjaunias' description, pediatric dural AV shunts can be classified into three different types: (1) dural sinus malformations, (2) infantile type DAVS, and (3) adult type DAVS [3].

Dural sinus malformations (DSMs) are rare lesions accounting for about $2 \%$ of pediatric vascular malformations which affect males more often (2:1). DSMs are not hereditary, and no known hereditary vascular disease, such as HHT is associated with them. They can be distinguished into those that involve the posterior sinuses, usually with giant dural lakes and slow-flow AV shunts and those involving the jugular bulb, which are associated with a high-flow sigmoid sinus AVF [4]. There are also some smaller sigmoid sinus-jugular bulb junction shunts that are seen at a slightly later age and believed to be induced later than the maturation of the jugular bulbs. DSM may lead to an abnormal development of the child's dural sinuses [4]. The natural course of the disease depends on the extent of the DSM, the location of the involved dural sinus, and the fistula flow $[4,5]$. Known good prognostic features are the involvement of a unilateral dural sinus without affection of the torcular herophili or the superior sagittal sinus, allowing the brain to drain using the other side. Other good characteristics are the absence of venous reflux into cortical veins or large sinuses and the absence of dysmaturation of the jugular bulb, therefore the patency of venous outlets [5]. The most common clinical presentations are macrocrania, seizures, and mental retardation.

While DSM mostly present with acute symptoms such as infarction, hemorrhage, or convulsions, infantile DAVSs show more delayed symptoms like mental retardation or progressive neurologic deterioration [5]. Infantile-type DAVS (IDAVSs) are the most frequent type of pediatric DAVs. They are high flow and low pressure. Typically, they consist of large and patent sinuses without lakes, are multifocal, and characteristically induce pial AV shunts. IDAVS are initially well tolerated, but long-term prognosis is poor with neurological deterioration in early adulthood. Adult type DAVS (ADAVS) are mostly located in the cavernous plexus [4].

\section{Langer-Giedion syndrome}

LGS is a very rare congenital disease; therefore, numbers of incidence and prevalence are difficult to determine. However, at least 100 cases have been reported in the literature so far. Long-term follow-ups and reports about natural clinical course and complications are rare $[1,6,7]$.

All patients show a de novo mutation involving 8q24.1, spanning the TRPS I and EXT1 genes. Therefore, the phenotype of the syndrome is a combination of two independent diseases caused by these deletions: tricho-rhino-phalangeal syndrome type I (TRPS I) and hereditary multiple exostoses (HME) [6].

There have been few reported cases of LGS with central nervous system abnormalities such as dysmorphism and hypoplasia of corpus callosum, septum pellucidum, or pituitary gland. These findings were associated with some new genetic changes, which have never been reported before in combination with deletion of 8q, causing LGS: deletion of 21q22.1 and balanced translocation $\mathrm{t}(2 ; 11)(\mathrm{p} 24 ; \mathrm{p} 15)[6]$. Up to $70 \%$ of patients with LGS show mild to severe cognitive disability [7]. The extent of $8 \mathrm{q}$ deletion is directly proportional to cognitive disability. RAD21, a gene located on chromosome $8 \mathrm{q}$, has been reported as possible candidate gene for brain anomalies such as dysplasia of corpus callosum [8]. Two patients were reported with brain anomalies such as enlargement of the lateral ventricles and T2w-hyperintensity of the tuber cinereum [7].

The fact that brain MRI has not been performed in every patient reported with LGS probably leads to an underestimation of associated brain anomalies [6] and could be the reason why intracranial $\mathrm{AV}$ fistulas never have been reported in these patients before.

\section{Our case}

In our case, the involvement of an embryological persistent artery, the pouch of the sigmoid sinus, the extensive recruitment of all meningeal arteries were considered convincing indicators of the congenital nature of the lesion. The absence of tinnitus as a presenting symptom can be explained because it was perceived as natural since it was present at birth. The shunt likely belongs to the DSM type, developed in a favorable location and after the jugular bulbs maturation. Thus, any constrain for the normal cerebral venous drainage was relatively well compensated especially after "cavernous sinus capture". Presumably, the clinical manifestations remained restricted to hydrodynamic symptoms expressed as macrocrania instead of microcephaly associated with LGS. The above can explain why the lesion remained asymptomatic for 20 years. The venous system, which grew together with the shunt and developed efficient collateral drainage, tolerated the lesion until the slow and gradual increase of flow and venous strain started disrupting the balance. The lack of multifocality and associated pial AV shunts, both features of the infantile type, were deemed as additional factors for the DSM type.

\section{Conclusion}

We report here a case of a patient with known LGS and complex congenital dural intracranial AV fistula. To our 
knowledge, the association of LGS with dural AV fistulas has not been reported before.

\section{References}

1. Langer LO, Krassikoff N, Laxova R, Scheer-Williams M, Lutter LD, Gorlin RJ, Jennings CG, Day DW (1984) The tricho-rhino-phalangeal syndrome with exostoses (or Langer-Giedion syndrome): four additional patients without mental retardation and review of the literature. Am J Med Genet 19:81-112

2. Giedion A, Kesztler R, Muggiasca F (1975) The widened spectrum of multiple cartilaginous exostosis (MCE). Pediatr Radiol 3:93-100

3. Lasjaunias P (1997) Vascular diseases in neonates, infants and children. Interventional Neuroradiology Management, Springer-Verlag, Berlin

4. Lasjaunias P, Magufis G, Goulao A, Piske R, Suthipongchai S, Rodesch R, Alvarez H (1996) Anatomoclinical aspects of dural arteriovenous shunts in children. Review of 29 cases. Interv Neuroradiol 2:179-191

5. Barbosa M, Mahadevan J, Weon YC, Yoshida Y, Ozanne A, Rodesch G, Alvarez H, Lasjaunias P (2003) Dural sinus malformations (DSM) with giant lakes, in neonates and infants. Review of 30 consecutive cases. Interv Neuroradiol 9:407-424

6. Cappuccio G, Genesio R, Ronga V, Casertano A, Izzo A, Riccio MP, Bravaccio C, Salerno MC, Nitsch L, Andria G, Melis D (2013) Complex chromosomal rearrangements causing Langer-Giedion syndrome atypical phenotype: genotype-phenotype correlation and literature review. Am J Med Genet A

7. Wuyts W, Roland D, Lüdecke HJ, Wauters J, Foulon M, Van Hul W, Van Maldergem L (2002) Multiple exostoses, mental retardation, hypertrichosis, and brain abnormalities in a boy with a de novo 8q24 submicroscopic interstitial deletion. Am J Med Genet $113: 326-332$

8. Halevy A, Basel-Vanagaite L, Shuper A, Helman S, Har-Zahav A, Birk E, Maya I, Kornreich L, Inbar D, Nürnberg G, Nürnberg P, Steinberg T, Straussberg R (2012) Microcephaly-thin corpus callosum syndrome maps to 8q23.2-q24.12. Pediatr Neurol 46: 363-368 